\title{
A RIM-METRIZABLE CONTINUUM
}

\author{
J. NIKIEL, L. B. TREYBIG, AND H. M. TUNCALI
}

(Communicated by Franklin D. Tall)

\begin{abstract}
A locally connected rim-metrizable continuum is constructed which admits a continuous mapping onto a non rim-metrizable space.
\end{abstract}

\section{INTRODUCTION}

We consider Hausdorff spaces and continuous mappings only. By a continuum we mean a compact and connected space. An arc is a linearly ordered continuum. It is well known that each separable arc is homeomorphic to $[0,1]$. Recall that a space $Y$ is said to be scattered if each nonempty subset of $Y$ has an isolated point. We shall say that a space $X$ is rim-metrizable (resp. rimcountable or rim-scattered) if $X$ has a basis $\mathscr{B}$ of open sets such that $\operatorname{bd}(U)$ is metrizable (resp. countable or scattered) for each $U \in \mathscr{B}$. It is well known that each compact and countable space is both scattered and metrizable. Hence, each rim-countable compact space is rim-metrizable and rim-scattered.

Of course, each 0-dimensional compact space is rim-metrizable. Therefore, it is interesting to investigate compact rim-metrizable spaces which are not 0 dimensional only. Then it is natural to restrict attention to continua. That restriction is not strong enough yet, and the most interesting problems arise when locally connected rim-metrizable continua are studied.

Our aim is to construct a continuum $X$ whose existence proves the following theorem:

Theorem 1. The continuous image of a locally connected and rim-metrizable continuum need not be a rim-metrizable space.

Theorem 1 provides the negative answer to a 1987 question of E. D. Tymchatyn. The desired continuum $X$ is constructed as the inverse limit of an $\omega_{1}$-long transfinite inverse sequence of copies of the square $[0,1]^{2}$ with carefully chosen bonding maps. The construction of $X$ and proofs of its properties are given in Section 3, while Section 2 contains auxiliary results. Recall here

Received by the editors February 18, 1993.

1991 Mathematics Subject Classification. Primary 54F15; Secondary 54B15, 54C05.

Key words and phrases. Continuum, locally connected, rim-metrizable, continuous image, inverse limit.

The third author was supported by the Natural Sciences and Engineering Research Council of Canada. 
that [11] contains a rather simple example of a rim-metrizable continuum which is not locally connected and can be mapped onto a non-rim-metrizable space.

In $1967 \mathrm{~S}$. Mardesič proved that each space which is a continuous image of an arc is rim-metrizable ([6], see also [7] and [5] for stronger results). Simple examples show that there exist locally connected rim-metrizable continua which are not continuous images of arcs. Rather complicated methods were employed in [8] in order to get a locally connected and rim-countable (whence: rimmetrizable) continuum which is the continuous image of no arc. Many nice results about rim-metrizable spaces were obtained in [9], [10] and [11]. In particular, the following Theorem 2 is related to our Theorem 1:

Theorem 2 [11, Theorem 3.5]. If $Y$ is a locally connected continuum which is the image of a rim-metrizable continuum under a pseudo-confluent mapping, then $Y$ is rim-metrizable.

Theorem 3 [11, Theorem 2.8]. A product $X \times Y$ of compact spaces $X$ and $Y$ is rim-metrizable if and only if one of the following conditions is satisfied:

(a) both $X$ and $Y$ are metrizable;

(b) both $X$ and $Y$ are 0-dimensional;

(c) one of $X$ and $Y$ is rim-metrizable and the other is metrizable and zerodimensional.

Since rim-metrizability is a hereditary property, Theorem 3 yields the following Lemma 1 which will be needed later.

Lemma 1. If a space $Z$ contains a subset homeomorphic to the product $X \times[0,1]$ of a non-metrizable compact space $X$ and $[0,1]$, then $Z$ is not rim-metrizable.

The reader is referred to [2] for general and well-known facts on inverse systems and their limits. More special properties of inverse limit spaces will be provided with appropriate references.

\section{AUXILIARY CONSTRUCTIONS}

Let $C$ be a copy of the Cantor set in the open interval $] 0,1[$. We shall consider $C$ with its linear ordering inherited from $] 0,1[$.

Let $Z$ be a subset of $[0,1]^{2}$ such that $Z$ is homeomorphic to $C \times[0,1]$. Let $B=(\{0,1\} \times[0,1]) \cup([0,1] \times\{0,1\})$ denote the boundary of the square $[0,1]^{2}$. We shall say that $Z$ is well-placed if $K \cap B$ consists of exactly two points which are the end points of $K$, for each component $K$ of $Z$.

Now, let $Z$ be a well-placed copy of $C \times[0,1]$ in $[0,1]^{2}$. Then $[0,1]^{2}-$ $K$ has exactly two components, for each component $K$ of $Z$. Let $h: Z \rightarrow$ $C \times[0,1]$ be a homomorphism and, for each component $K$ of $Z$, let $c_{K} \in$ $C$ be such that $h(K)=\left\{c_{K}\right\} \times[0,1]$. We shall say that $h$ is a placement homeomorphism if $h(Z \cap M)=\left\{c \in C: c<c_{K}\right\} \times[0,1]$ or $h(Z \cap M)=\{c \in$ $\left.C: c>c_{K}\right\} \times[0,1]$ for each component $K$ of $Z$ and each component $M$ of $[0,1]^{2}-K$.

We omit proofs of the following two lemmas. Lemma 2 is quite trivial, and proofs of results similar to Lemma 3 can be found in [3].

Lemma 2. If $Z$ is a well-placed copy of $C \times[0,1]$ in $[0,1]^{2}$, then there exists a placement homeomorphism $h: Z \rightarrow C \times[0,1]$. 
Lemma 3. If $Z$ is a well-placed copy of $C \times[0,1]$ in $[0,1]^{2}$ and $h: Z \rightarrow$ $C \times[0,1]$ is a placement homeomorphism, then $h$ can be extended to a homeomorphism $H:[0,1]^{2} \rightarrow[0,1]^{2}$.

Let $A$ be a subset of cardinality $\aleph_{1}$ of ]0,1[ such that the complement of $A$ is dense in $[0,1]$ (the latter assumption is going to simplify some arguments in the next section). Let $\left\{a_{\alpha}: \alpha<\omega_{1}\right\}$ be an enumeration of $A$.

Let $L=([0,1] \times\{0\}) \cup(A \times[0,1])$ and order $L$ lexicographically; i.e., let $\langle x, y\rangle<\left\langle x^{\prime}, y^{\prime}\right\rangle$ if either $x<x^{\prime}$ or $x=x^{\prime}$ and $y<y^{\prime}$. Then $<$ is a linear ordering on $L$. We consider $L$ with its order topology introduced by the subbasis of intervals of the form $\{u: u<v\}$ or $\{u: v<u\}, v \in$ $L-\{\langle 0,0\rangle,\langle 1,0\rangle\}$, for open sets. Then $L$ is an ordered continuum (三 a Hausdorff arc) which is nonseparable and, hence, nonmetrizable.

Define $r: L \rightarrow[0,1]$ by $r(\langle x, y\rangle)=x$. Then $r$ is a continuous onto map and $r^{-1}(x)$ is a nondegenerate closed sub-arc of $L$ for each $x \in A$. For every $\alpha<\omega_{1}$ let $I_{\alpha}=r^{-1}\left(a_{\alpha}\right)$. Hence, each $I_{\alpha}$ is a copy of $[0,1]$ and $I_{\alpha} \cap I_{\alpha^{\prime}}=\varnothing$ if $\alpha \neq \alpha^{\prime}$.

For $\alpha<\omega_{1}$, let $\mathscr{G}_{\alpha}$ denote the decomposition of $L$ into the arcs $I_{\beta}, \alpha \leq$ $\beta<\omega_{1}$, and points. Since each decomposition of an arc into sub-arcs and points is upper semicontinuous, the quotient space $L_{\alpha}=L / \mathscr{G}_{\alpha}$ is a Hausdorff arc again. Let $r_{\alpha}: L \rightarrow L_{\alpha}$ denote the quotient map.

Observe that $L_{0}=[0,1]$ and $r_{0}=r$. Also, each arc $L_{\alpha}, \alpha<\omega_{1}$, is separable and therefore homeomorphic to $[0,1]$.

Now, suppose that $\alpha \leq \beta \leq \gamma<\omega_{1}$. Then $\mathscr{G}_{\beta}$ refines $\mathscr{G}_{\alpha}$. Hence, there is the unique $r_{\alpha}^{\beta}: L_{\beta} \rightarrow L_{\alpha}$ such that $r_{\alpha}=r_{\alpha}^{\beta} \circ r_{\beta}$. Clearly, $r_{\alpha}^{\beta}$ is continuous, monotone, and onto. Moreover, $r_{\alpha}^{\gamma}=r_{\alpha}^{\beta} \circ r_{\beta}^{\gamma}$. Thus, we obtain an inverse system $\mathscr{R}=\left(L_{\alpha}, r_{\alpha}^{\beta}, \alpha \leq \beta<\omega_{1}\right)$ of metrizable arcs $L_{\alpha}$ with monotone onto bounding maps $r_{\alpha}^{\beta}$.

Note that $L$ is canonically homeomorphic to $\lim$ inv $\mathscr{R}$. Furthermore, if $\lambda$ is a limit ordinal number with $\lambda<\omega_{1}$, then $\mathscr{R}_{\lambda}=\left(L_{\alpha}, r_{\alpha}^{\beta}, \alpha \leq \beta<\lambda\right)$ is an inverse system such that $L_{\lambda}$ is canonically homeomorphic to lim inv $\mathscr{R}_{\lambda}$. We identify $L$ with lim inv $\mathscr{R}$ and each $L_{\lambda}$ with lim inv $\mathscr{R}_{\lambda}$.

It is convenient to introduce the following notation. For each $\alpha<\omega_{1}$ let $s_{\alpha}=\operatorname{id}_{C} \times r_{\alpha}$ denote the product map of $C \times L$ onto $C \times L_{\alpha}$, i.e., $s_{\alpha}(c, u)=$ $\left(c, r_{\alpha}(u)\right)$ for all $c \in C$ and $u \in L$. Furthermore, for every $\alpha \leq \beta<\omega_{1}$, let $s_{\alpha}^{\beta}=\operatorname{id}_{C} \times r_{\alpha}^{\beta}: C \times L_{\beta} \rightarrow C \times L_{\alpha}$. Obviously, $\mathscr{S}=\left(C \times L_{\alpha}, s_{\alpha}^{\beta}, \alpha \leq \beta<\omega_{1}\right)$ is an inverse system such that lim inv $\mathscr{S}=C \times L$.

\section{The MAIN RESUlt}

We are going to apply transfinite induction to construct spaces $X_{\alpha}$ and $Z_{\alpha}$, $\alpha<\omega_{1}$, and mappings $t_{\alpha}^{\beta}: X_{\beta} \rightarrow X_{\alpha}, \alpha \leq \beta<\omega_{1}$, and $h_{\alpha}: C \times L_{\alpha} \rightarrow Z_{\alpha}$, $\alpha<\omega_{1}$, such that the following properties (1)-(6) are satisfied for all $0 \leq \alpha \leq$ $\beta \leq \gamma<\omega_{1}$ :

(1) $X_{\alpha}=[0,1]^{2}$

(2) $Z_{\alpha} \subset X_{\alpha}$ and $Z_{\alpha}=C \times[0,1]$

(3) $h_{\alpha}$ is a homeomorphism of $C \times L_{\alpha}$ onto $Z_{\alpha}=C \times[0,1]$ such that the first coordinate of each point $h_{\alpha}(c, u)$ is $c$;

(4) $t_{\alpha}^{\beta}\left(Z_{\beta}\right)=Z_{\alpha}$ and $\left(\left.t_{\alpha}^{\beta}\right|_{\beta}\right) \circ h_{\beta}=h_{\alpha} \circ s_{\alpha}^{\beta}$; 
(5) $t_{\alpha}^{\beta}\left(X_{\beta}-Z_{\beta}\right)=X_{\alpha}-Z_{\alpha}$ and $\left.t_{\alpha}^{\beta}\right|_{X_{\beta}-Z_{\beta}}: X_{\beta}-Z_{\beta} \rightarrow X_{\alpha}-Z_{\alpha}$ is a homeomorphism;

(6) $t_{\alpha}^{\alpha}=\mathrm{id}_{X_{\alpha}}$ and $t_{\alpha}^{\gamma}=t_{\alpha}^{\beta} \circ t_{\beta}^{\gamma}$.

Let $X_{0}=[0,1]^{2}, Z_{0}=C \times[0,1]=C \times L_{0}$, and $h_{0}=\mathrm{id}_{Z_{0}}$.

Suppose that for some ordinal number $\delta$ with $0<\delta<\omega_{1}$, the required spaces $X_{\alpha}$ and $Z_{\alpha}, \alpha<\delta$, and mappings $t_{\alpha}^{\beta}, \alpha \leq \beta<\delta$, and $h_{\alpha}, \alpha<\omega_{1}$, are already constructed.

First, consider the case when $\delta=\varepsilon+1$. Let $X_{\delta}=[0,1]^{2}$ and $Z_{\delta}=C \times$ $[0,1] \subset X_{\delta}$. Let $\mathscr{F}$ denote the decomposition of $X_{\delta}$ into the arcs $\{c\} \times\left[\frac{1}{3}, \frac{2}{3}\right]$, $c \in C$, and points. Also, let $f: X_{\delta} \rightarrow X_{\delta} / \mathscr{F}$ denote the quotient map. Since $X_{\delta} / \mathscr{F}$ is homeomorphic to the square, we may let $i: X_{\delta} / \mathscr{F} \rightarrow[0,1]^{2}$ be a homeomorphism. Clearly, $i\left(f\left(Z_{\delta}\right)\right)$ is a well-placed copy of $C \times[0,1]$.

Recall that there is exactly one point in $L_{\varepsilon}$ whose pre-image under $r_{\varepsilon}^{\delta}$ is nondegenerate, and this point is not an end point of $L_{\varepsilon}$. Furthermore, $s_{\varepsilon}^{\delta}=$ $\operatorname{id}_{C} \times r_{\varepsilon}^{\delta}$. Therefore, there exist homeomorphisms $h_{\delta}: C \times L_{\delta} \rightarrow Z_{\delta}$ and $j:$ $i\left(f\left(Z_{\delta}\right)\right) \rightarrow Z_{\varepsilon}$ such that the first coordinate of each point $h_{\delta}(c, u)$ is $c$ and $h_{\varepsilon} \circ s_{\varepsilon}^{\delta}=j \circ i \circ f \circ h_{\delta}$. Clearly, $j$ must be a placement homeomorphism. By Lemma 3, there exists a homeomorphism $J: i\left(f\left(X_{\delta}\right)\right)=[0,1]^{2} \rightarrow X_{\varepsilon}=[0,1]^{2}$ which extends $j$. It suffices to let $t_{\varepsilon}^{\delta}=J \circ i \circ f$ and $t_{\alpha}^{\delta}=t_{\alpha}^{\varepsilon} \circ t_{\varepsilon}^{\delta}$ for each $\alpha \leq \varepsilon$.

Now, consider the case when $\delta$ is a limit ordinal number, $0<\delta<\omega_{1}$. By the inductive assumptions (6) and (4), $\left(X_{\alpha}, t_{\alpha}^{\beta}, \alpha \leq \beta<\delta\right)$ and $\left(Z_{\alpha},\left.t_{\alpha}^{\beta}\right|_{z_{\beta}}, \alpha\right.$ $\leq \beta<\delta$ ) are well-defined inverse systems. Let $X_{\delta}^{\prime}$ and $Z_{\delta}^{\prime}$ denote their inverse limits, respectively. Then $Z_{\delta}^{\prime} \subset X_{\delta}^{\prime}$. Also, let $\pi_{\alpha}: X_{\delta}^{\prime} \rightarrow X_{\alpha}, \alpha<\delta$, denote the natural projections and let $h_{\delta}^{\prime}: C \times L_{\delta} \rightarrow Z_{\delta}$ be the homeomorphism induced by the homeomorphisms $h_{\alpha}, \alpha<\delta$.

For any sequence $\left(\varepsilon_{n}\right)_{n=1}^{\infty}$ of ordinals which increases to $\delta$, there exists the natural homeomorphism of $X_{\delta}^{\prime}$ onto the inverse limit space $\lim \operatorname{inv}\left(X_{\varepsilon_{n}}, t_{\varepsilon_{n}}^{\varepsilon_{n+1}}\right)$ of the inverse sequence $\left(X_{\varepsilon_{n}}, t_{\varepsilon_{n}}^{\varepsilon_{n+1}}\right)$. Since the spaces $X_{\varepsilon_{n}}$ are copies of the square and all the maps $t_{\varepsilon_{n}}^{\varepsilon_{n+1}}$ are monotone, $X_{\delta}^{\prime}$ is homeomorphic to the square (see [1, Theorem 4 and the Corollary following it on p. 482]). Let $i: X_{\delta}^{\prime} \rightarrow$ $[0,1]^{2}$ be a homeomorphism. We already know that $Z_{\delta}^{\prime}$ is homeomorphic to $C \times L_{\delta}$ and so to $C \times[0,1]$. A simple direct proof shows that $i\left(Z_{\delta}^{\prime}\right)$ is a well-placed copy of $C \times[0,1]$. Hence there exists a homeomorphism $j:[0,1]^{2} \rightarrow[0,1]^{2}$ such that $j\left(i\left(Z_{\delta}^{\prime}\right)\right)=C \times[0,1]$ and the first coordinate of $j \circ i \circ h_{\delta}^{\prime}(c, u)$ is $c$ for each $(c, u) \in C \times L_{\delta}$.

It suffices to let $X_{\delta}=j\left(i\left(X_{\delta}^{\prime}\right)\right)=[0,1]^{2}, Z_{\delta}=j\left(i\left(Z_{\delta}^{\prime}\right)\right)=C \times[0,1], h_{\delta}=$ $j \circ i \circ h_{\delta}^{\prime}: C \times L_{\delta} \rightarrow Z_{\delta}$, and $t_{\alpha}^{\delta}=\pi_{\alpha} \circ i^{-1} \circ j^{-1}: X_{\delta} \rightarrow X_{\alpha}$ for each $\alpha<\delta$. This concludes the inductive construction.

By (6), $\left(X_{\alpha}, t_{\alpha}^{\beta}, \alpha \leq \beta<\omega_{1}\right)$ is an inverse system. We let $X$ denote its inverse limit. Then $X$ is a continuum as the inverse limit of continua. Since all the factor spaces $X_{\alpha}$ are locally connected continua and all the bonding maps $t_{\alpha}^{\beta}$ are monotone and onto, $X$ is a locally connected continuum (see, e.g., [4]). Thus we have the following properties of $X$ :

Claim 1. $X$ is a locally connected continuum.

Now, let us prove two more special properties of $X$.

Claim 2. $X$ is rim-metrizable. 
Let $t_{\alpha}: X \rightarrow X_{\alpha}, \alpha<\omega_{1}$, denote the projections. If $\mathscr{B}_{\alpha}$ is a basis of $X_{\alpha}$, for $\alpha<\omega_{1}$, then the collection $\mathscr{B}$ of all sets $t_{\alpha}^{-1}(U), U \in \mathscr{B}_{\alpha}, \alpha<\omega_{1}$, is a basis of $X$. For each $\alpha$, we are going to find a basis $\mathscr{B}_{\alpha}$ of $X_{\alpha}$ such that if $U \in \mathscr{B}_{\alpha}$, then $t_{\alpha}^{-1}(U)$ has metrizable boundary. Then $\mathscr{B}$ as above will be a basis of $X$ which consists of open sets with metrizable boundaries.

Let $\alpha<\omega_{1}$. Let $P_{\alpha}=\left\{x \in X_{\alpha}: t_{\alpha}^{-1}(x)\right.$ is nondegenerate $\}$. If $x \in P_{\alpha}$, then $x \in Z_{\alpha}$ and $s_{\alpha}^{-1}\left(h_{\alpha}^{-1}(x)\right)$ is nondegenerate, because $s_{\alpha}^{-1}\left(h_{\alpha}^{-1}(x)\right)=I_{\beta}$ for some $\beta$ such that $\alpha \leq \beta<\omega_{1}$. It follows that $h_{\alpha}^{-1}\left(P_{\alpha}\right)$ is a subset of $C \times L_{\alpha}$ which is contained in $C \times M_{\alpha}$, where $M_{\alpha}$ is the set of all points $\left(r_{0}^{\alpha}\right)^{-1}\left(a_{\beta}\right), \alpha \leq \beta<\omega_{1}$. Since $[0,1]-A$ is dense in $[0,1]$, the set $M_{\alpha}$ is 0-dimensional. By Lemma 3, we may assume that $P_{\alpha} \subset C \times N_{\alpha}$ for some 0 -dimensional subset $N_{\alpha}$ of $[0,1]$. Now, let $\mathscr{B}_{\alpha}$ be a countable basis of $X_{\alpha}=[0,1]^{2}$ such that $\operatorname{bd}(U) \cap\left(C \times N_{\alpha}\right)=\varnothing$ for each $U \in \mathscr{B}_{\alpha}$. Let $U \in \mathscr{B}_{\alpha}$, and observe that $\operatorname{bd}\left(t_{\alpha}^{-1}(U)\right)=t_{\alpha}^{-1}(\operatorname{bd}(U))$ and $t_{\alpha}$ is one-to-one on the set $\operatorname{bd}\left(t_{\alpha}^{-1}(U)\right)$. Hence, $t_{\alpha}$ maps $\operatorname{bd}\left(t_{\alpha}^{-1}(U)\right)$ homeomorphically onto $\operatorname{bd}(U) \subset$ $[0,1]^{2}$, and so $\operatorname{bd}\left(t_{\alpha}^{-1}(U)\right)$ is metrizable. This completes the proof of Claim 2.

Claim 3. $X$ can be mapped onto a space which is not rim-metrizable.

Let

$$
Z=\operatorname{liminv}\left(Z_{\alpha},\left.t_{\alpha}^{\beta}\right|_{\beta}, \alpha \leq \beta<\omega_{1}\right)=\bigcap_{\alpha<\omega_{1}} t_{\alpha}^{-1}\left(Z_{\alpha}\right) \subset X .
$$

The homeomorphisms $h_{\alpha}, \alpha<\omega_{1}$, induce the homeomorphism $h: C \times L \rightarrow$ $Z$.

Let $f$ be any mapping of the Cantor set $C$ onto $[0,1]$. Define $g: C \times L \rightarrow$ $[0,1] \times L$ by $g(c, u)=(f(c), u)$.

Let $\mathscr{G}$ be the decomposition of $X$ into the sets $h\left(g^{-1}(w)\right), w \in[0,1] \times L$, and points. Then $\mathscr{G}$ is upper semicontinuous, whence the quotient space $X / \mathscr{G}$ is Hausdorff. Observe that $X / \mathscr{G}$ contains a subset homeomorphic to $[0,1] \times L$. Since $L$ is non-metrizable, $X / \mathscr{G}$ is not rim-metrizable, by Lemma 1 .

\section{REMARKS}

1. The spaces $X$ and $Z$ were constructed by means of monotone mappings and inverse limits. However, the decomposition $\mathscr{G}$ in the proof of Claim 3 is not monotone. Furthermore, the construction cannot be modified to provide a monotone decomposition $\mathscr{G}^{\prime}$ with all the required properties. Indeed, by Theorem 2, a monotone image of a locally connected rim-metrizable continuum is rim-metrizable again.

2. Let $M$ be a subset of $X$ which consists of all points $y$ such that either $y=f_{0}^{-1}(x)$ for some $\left.x \in\right] 0,1[-(C \times[0,1])$ or $y=h(c, u)$ for some $c \in C$ and $u \in L$ such that $u \in \bigcup_{\alpha<\omega_{1}} I_{\alpha}=r^{-1}(A)$ and $u$ is not an end point of any $I_{\alpha}$. One can show that $M$ is a Hausdorff 2-manifold, i.e., each point of $M$ has an open neighborhood homeomorphic to $] 0,1{ }^{2}$. Also, $M$ is a $T_{3 \frac{1}{2}}$-space which is not normal.

3. The space $X$ we constructed above is 2-dimensional. It contains a closed subspace $Y$ such that $Y$ is a locally connected curve which has a basis of open sets with metrizable 0 -dimensional boundaries and yet $Z \subset Y$, whence continuous images of $Y$ need not be rim-metrizable. 
In fact, let $S$ be a copy of the Sierpinski universal plane curve such that $C \times[0,1] \subset S \subset[0,1]^{2}=X_{0}$ and let $Y=t_{0}^{-1}(S)$. By [12], it easily follows that $\left(t_{0}^{\alpha}\right)^{-1}(S)$ is a copy of $S$ contained in $X_{\alpha}, \alpha<\omega_{1}$. A modification of the proof of Claim 2 provides a basis of $Y$ as needed.

4. Continuous images of rim-countable continua and locally connected rimscattered continua were considered in [10], where it was proved that they can contain no subset homeomorphic to the product of a nonmetric compact space and a perfect set. It would be interesting to know if continuous images of locally connected rim-countable continua must be rim-metrizable. Also, it is unknown if one can find a perfectly normal space which is not rim-metrizable and is the continuous image of a locally connected rim-metrizable continuum. Some other questions concerning rim-properties of continua can be found in [8, p. 85]. In particular, it is asked there (see also [7]) if a locally connected rim-scattered continuum must be rim-countable.

\section{REFERENCES}

1. M. Brown, Some applications of an approximation theorem for inverse limits, Proc. Amer. Math. Soc. 11 (1960), 478-483.

2. R. Engelking, General topology, PWN, Warsaw, 1977.

3. R. J. Fokkink and L. G. Oversteegen, The geometry of laminations (in preparation).

4. G. R. Gordh, Jr., and S. Mardešić, Characterizing local connectedness in inverse limits, Pacific J. Math. 58 (1975), 411-417.

5. J. Grispolakis, J. Nikiel, J. N. Simone, and E. D. Tymchatyn, Separators in continuous images of ordered continua and hereditarily locally connected continua, Canad. Math. Bull. (to appear).

6. S. Mardešić, Images of ordered compacta are locally peripherally metric, Pacific J. Math. 23 (1967), 557-568.

7. J. Nikiel, H. M. Tuncali, and E. D. Tymchatyn, On the rim-structure of continuous images of ordered compacta, Pacific J. Math. 149 (1991), 145-155.

8. $\quad$ A locally connected rim-countable continuum which is the continuous image of no arc, Topology Appl. 42 (1991), 83-93.

9. H. M. Tuncali, Some generalizations of the Hahn-Mazurkiewicz theorem, Ph.D. Thesis, University of Saskatchewan, Saskatoon, 1989.

10. _ Analogues of Treybig's product theorem, Proc. Amer. Math. Soc. 108 (1990), 855858.

11. Concerning continuous images of rim-metrizable continua, Proc. Amer. Math. Soc. 113 (1991), 461-470.

12. G. T. Whyburn, Topological characterization of the Sierpinski curve, Fund. Math. 45 (1958), 320-324.

(J. Nikiel) Department of Mathematics, American University of Beirut, Beirut, LebaNON

E-mail address: nikiel@layla.aub.ac.lb

(L. B. Treybig) Department of Mathematics, Texas A\&M University, College Station, TEXAS 77843-3368

E-mail address: treybig@math.tamu.edu

(H. M. Tuncali) College of Arts and Sciences, Nipissing University College, North Bay, Ontario, Canada P1B 8L7

E-mail address: muratt@einstein.unipissing.ca 